\title{
Security on cloud and pass
}

\author{
Mr Karthik.B ${ }^{1}$, Mrs Devi selvam² \\ Student, Computer Science and Engineering, Sri Shakthi institute of Engineering and technology, Coimbatore, India ${ }^{1}$ \\ Assistant Professor, Computer Science and Engineering, Sri Shakthi institute of Engineering and technology, \\ Coimbatore, India ${ }^{2}$
}

\begin{abstract}
The term "cloud computing" has been mentioned for just under two years in relation to services or infrastructural resources, which can be contracted over a network. Cloud computing is a flexible, cost-effective, and proven delivery platform for providing business or consumer it services over the internet. However, cloud computing presents an added level of risk because essential services are often outsourced to a third party, which makes it harder to maintain data security and privacy, support data, service availability and demonstrate compliance. we analyze many technologies, it also inherits their security issues identifying the main vulnerabilities in this kind of systems and the most important threats found in the literature related to cloud computing and its environment as well as to identify and relate vulnerabilities and threats with possible solutions. Cloud computing is surrounded by many security issues like securing data, and examining the utilization of cloud by the cloud computing vendors. Cloud computing has brought lots of security challenges for the consumers and service providers. The study aims to identify the most vulnerable security threats in cloud computing, which will enable both end users and vendors to know about the key security threats associated with cloud computing. It will enable researchers and security professionals to know about users, vendors concerns and also to critical analysis about the different security models and tools proposed.
\end{abstract}

Keywords: Cloud security, Iaas, Paas, cloud computing

\section{INTRODUCTION}

To Implement the security in cloud environment we have to review more new techniques and current cloud scenarios and how's the developer and client has communicate and use the services provide by cloud then we analysis the working model of cloud with specific cloud providers like amazon or other open source cloud provides like open stack, cloud stack then the application and web service which has deployed in each cloud services.

Deployment of complete cloud product will have integration of all layers in cloud service like IAAS, PASS, SAAS, web Service and Web application. So we have to analysis and the basic networks and virtual network, virtual hosting, Virtual machine and network concepts.

In cloud computing we have multiple users and server communicate simultaneously through internet mainly in remote commutation. So there is possible of leakage of security by identify and verification of authorized user to the particular service to the correct user so we focus on authentication and authorization.

In web services and application commonly for authentication and authorized we using the authorized keys, token, user and password id's which is used to make secure login and provide access control to the user to the correct privileges. To improve the security in cloud computing we analysis the current technology like multitenancy, OAuth 2.0 , Identity verification service, Single sign-on (SSO),Open ID , SAML, Kerberos ,ws-trust , XML signature, secure shell.

In cloud computing resource sharing and centralized database and storage concept has been played the main role.so we also focus on the storage to avoid the loss of data in the database and attack on database by unauthorized users then one single data will be used by more than one user in the same time so there is the possible of damage or corrupt of data in the database .to improve the security in storage concept we have to use Data replication and failover concept to make cloud storage as secure and flexible.

To provide the security on cloud and PAAS we have to implement the cloud environment. The cloud environment have 5 layers also called as modules. The overall security Architecture has been figure out below Fig 3.1.

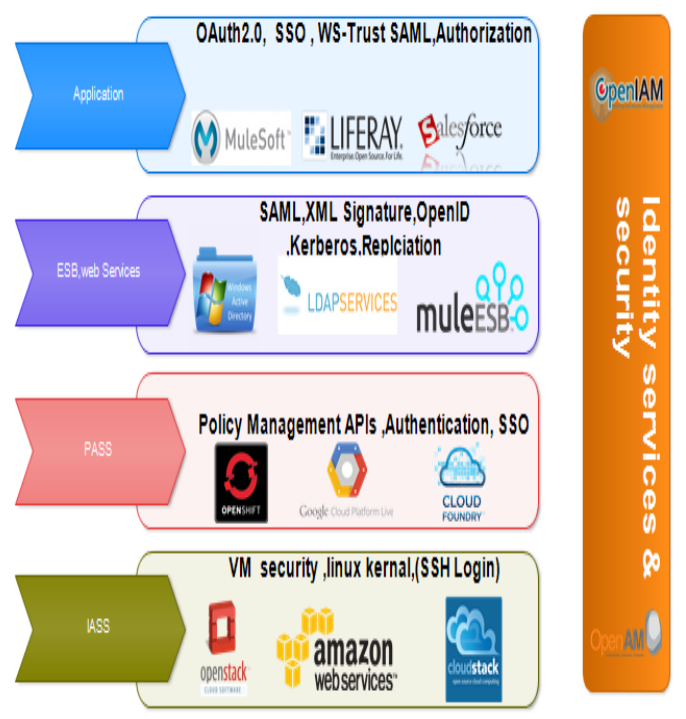

Fig 3.1 Cloud security Architecture 


\section{IAAS: INFRASTRUCTURE AS A SERVICE}

Cloud infrastructure services, known as "Infrastructure as a Service"(IaaS), deliver computer infrastructure (such as a platform virtualization environment), storage, and networking. Instead of having to purchase software, servers, or network equipment, users can buy these as a fully outsourced service that is usually billed according to the amount of resources consumed.

There are multiple IAAS service providers like openstack, amazon,cloudstack, etc .we have implement IAAS by using the cloud stack

\section{APACHE CLOUDSTACK}

Apache CloudStack[9] is open source software designed to deploy and manage large networks of virtual machines, as a highly available, highly scalable Infrastructure as a Service (IaaS) cloud computing platform. CloudStack is used by a number of service providers to offer public cloud services, and by many companies to provide an onpremises (private) cloud offering, or as part of a hybrid cloud solution.

CloudStack is a turnkey solution that includes the entire "stack" of features most organizations want with an IaaS cloud: compute orchestration, Network-as-a-Service, user and account management, a full and open native API, resource accounting, and a first-class User Interface (UI). CloudStack[10] currently supports the most popular hypervisors: VMware, KVM, XenServer, Xen Cloud Platform (XCP) and Hyper-V.

Users can manage their cloud with an easy to use Web interface, command line tools, and/or a full-featured RESTful API. In addition, CloudStack provides an API that's compatible with AWS EC2 and S3 for organizations that wish to deploy hybrid clouds.

Use CloudStack to deploy, manage, and configure cloud computing environments.

Typical users are service providers and enterprises. With CloudStack, you can:

- $\quad$ Set up an on-demand, elastic cloud computing service. Service providers can sell self-service virtual machine instances, storage volumes, and networking configurations over the Internet.

- $\quad$ Set up an on-premise private cloud for use by employees. Rather than managing virtual machines in the same way as physical machines, with CloudStack an enterprise can offer self-service virtual machines to users without involving IT departments.

\section{CLOUDSTACK USAGE}

\section{Multiple Hypervisor Support}

CloudStack works with a variety of hypervisors, and a single cloud deployment can contain multiple hypervisor implementations. The current release of CloudStack supports pre-packaged enterprise solutions like Citrix XenServer and VMware vSphere, as well as KVM or Xen running on Ubuntu or CentOS.

\section{Massively Scalable Infrastructure Management}

CloudStack can manage tens of thousands of servers installed in multiple geographically distributed datacenters. The centralized management server scales linearly, eliminating the need for intermediate cluster-level management servers. No single component failure can cause cloud-wide outage. Periodic maintenance of the management server can be performed without affecting the functioning of virtual machines running in the cloud.

\section{Automatic Configuration Management}

CloudStack automatically configures each guest virtual machine's networking and storage settings. CloudStack internally manages a pool of virtual appliances to support the cloud itself. These appliances offer services such as firewalling, routing, DHCP, VPN access, console proxy, storage access, and storage replication. The extensive use of virtual appliances simplifies the installation, configuration, and ongoing management of a cloud deployment.

\section{Graphical User Interface}

CloudStack offers an administrator's Web interface, used for provisioning and managing the cloud, as well as an end-user's Web interface, used for running VMs and managing VM templates. The UI can be customized to reflect the desired service provider or enterprise look and feel.

\section{API and Extensibility}

CloudStack provides an API that gives programmatic access to all the management features available in the UI. The API is maintained and documented. This API enables the creation of command line tools and new user interfaces to suit particular needs.

\section{High Availability}

CloudStack has a number of features to increase the availability of the system. The Management Server itself may be deployed in a multi-node installation where the servers are load balanced. MySQL may be configured to use replication to provide for a manual failover in the event of database loss. For the hosts, CloudStack supports NIC bonding and the use of separate networks for storage as well as iSCSI Multipath.

\section{PAAS-PLATFORM AS A SERVICE}

The most complex of the three, cloud platform services or "Platform as a Service"(PaaS) deliver computational resources through a platform. What developers gain with PaaS is a framework they can build upon to develop or customize applications. PaaS makes the development, testing, and deployment of applications quick, simple, and cost-effective, eliminating the need to buy the underlying layers of hardware and software.

There are multiple paas providers are available like Google App Engine, Heroku, Windows Azure Cloud Services, Amazon AWS, Red Hat OpenShift . We have deploy Red Hat Open shift on

IAAS (Infrastructure as a Service). And also we can deploy Red Hat Open shift in separate service in organization server and provide services and application 


\section{OPENSHIFT}

OpenShift[11] is Red Hat's Cloud Computing Platform as a Service (PaaS) offering. OpenShift is an application platform in the cloud where application developers and teams can build, test, deploy, and run their applications.

OpenShift takes care of all the infrastructure, middleware, and management and allows the developer to focus on what they do best: designing and coding applications.

OpenShift takes a No-Lock-In approach to PaaS by providing built-in support for Node.js, Ruby, Python, PHP, Perl, and Java (the standard in today's Enterprise). In addition, OpenShift is extensible with a customizable cartridge functionality that allows enterprising developers to add any other language they wish. We've seen everything from Clojure to COBOL running on OpenShift. In addition to this flexible, no-lock-in, language approach, OpenShift supports many of the popular frameworks that make a developer's life easier including frameworks ranging from spring, to Rails, to Play. OpenShift is designed to allow Developers to work the way they want to work by giving them the languages, frameworks and tools they need for fast and easy application development. OpenShift takes care of maintaining the services underlying the application and scaling the application as needed[12].

\begin{tabular}{ll}
\multicolumn{2}{l}{ SUPPORTED LANGUAGE ENVIRONMENTS } \\
$>$ & JAVASCRIPT \\
$>$ & RUBY \\
$>$ & PYTHON \\
$>$ & PHP \\
$>$ & PERL \\
$>$ & JAVA \\
$>$ & HASKELL \\
$>$ & .NET
\end{tabular}

$\begin{array}{ll}\text { SUPPORTED DATABASES } \\ > & \text { MYSQL } \\ > & \text { POSTGRESQL } \\ > & \text { MONGODB } \\ > & \text { MICROSOFT SQL SERVER }\end{array}$

\section{SUPPORTED FRAMEWORKS}

OpenShift supports web-application frameworks by supporting each language's preferred web-integration API, with no required changes to the actual framework code.

$$
\begin{array}{ll}
> & \text { Rack for Ruby } \\
> & \text { WSGI for Python } \\
> & \text { PSGI for Perl } \\
& \text { Node.js for JavaScript }
\end{array}
$$

WEB SERVICE \& ENTERPRISE SERVICE BUS (ESB)

Web services are client and server applications that communicate over the World Wide Web's (WWW) Hypertext Transfer Protocol (HTTP). As described by the World Wide Web Consortium (W3C), web services provide a standard means of interoperating between software applications running on a variety of platforms and frameworks. Web services are characterized by their great interoperability and extensibility, as well as their machine-process able descriptions, thanks to the use of XML. Web services can be combined in a loosely coupled way to achieve complex operations. Programs providing simple services can interact with each other to deliver sophisticated added-value services

An enterprise service bus (ESB) is a software architecture model used for designing and implementing communication between mutually interacting software applications in a service-oriented architecture (SOA). As a software architectural model for distributed computing it is a specialty variant of the more general client server model and promotes agility and flexibility with regard to communication between applications. Its primary use is in enterprise application integration (EAI) of heterogeneous and complex landscapes.

We Implement the OpenLDAP to Integrate with the ESB and Web service to monitor and manage the user and services in the cloud services.

\section{OPENLDAP}

OpenLDAP [5] is an open source implementation of the Lightweight Directory Access Protocol (LDAP) developed by the OpenLDAP Project. It is released under its own BSD-style license called the OpenLDAP Public License .LDAP is a platform-independent protocol.

LDAP stands for Lightweight Directory Access Protocol. As the name suggests, it is a lightweight protocol for accessing directory services, specifically X.500-based directory services. LDAP runs over TCP/IP or other connection oriented transfer services

Benefit of using LDAP is that information for an entire organization can be consolidated into a central repository. For example, rather than managing user lists for each group within an organization, LDAP can be used as a central directory accessible from anywhere on the network. And because LDAP supports Secure Sockets Layer (SSL) and Transport Layer Security (TLS), sensitive data can be protected from prying eyes.

LDAP also supports a number of back-end databases in which to store directories. This allows administrators the flexibility to deploy the database best suited for the type of information the server is to disseminate. Because LDAP also has a well-defined client Application Programming Interface (API), the number of LDAP-enabled applications are numerous and increasing in quantity and quality.

\section{WEB APPLICATION}

A web application or web app is any software that runs in a web browser. It is created in a browser-supported programming language (such as the combination of JavaScript, HTML and CSS) and relies on a web browser to render the application. Web applications are popular due to the ubiquity of web browsers, and the convenience of using a web browser as a client, sometimes called a thin client. The ability to update and maintain web applications without distributing and installing software on potentially thousands of client computers is a key reason for their popularity, as is the inherent support for cross-platform compatibility. Common web applications include 
webmail, online retail sales, online auctions, wikis and internet. It has the potential to make an increasingly many other functions. We Implement Wso2, Liferay and significant contribution to economic activity throughout OpenIAM to provide security and access management and the world. This potential will only be realized if cloud Identity verification service among the web services, computing products and services are portable and PAAS, IAAS.

\section{LIFERAY}

Liferay[14] is a free and open-source enterprise portal written in Java and distributed under the GNU LGPL. Now in its eleventh year of development, the awardwinning product is one of the most widely deployed portal technologies on the market, with an estimated 250,000 deployments worldwide. More than a portal, Liferay is a platform for creating effective business applications and solutions. It offers a robust feature set, impressive scalability, time-saving development tools, support for over 30 languages, and a flexible, scalable architecture that is open-source developed and enterprise refined.

$>\quad$ Created in 2000 and open sourced in 2002.

$>\quad$ First portal to introduce Web OS

$>$

$>\quad$ Members of the JSR 286 (Portlet Specification)

\& JSR 314 (Java ServerFaces Specifications) Committees

Liferay Portal is the world's leading open source enterprise portal solution using the latest in Java and Web 2.0 technologies.

$>\quad$ Built in Content Management System (CMS) and Collaboration Suite

$>\quad$ usability - choose from over 60 portlets and over 20 plus themes

$>$ development tools

$>\quad$ Runs on all major application servers, databases and operating systems (over 700 deployment configurations)

$>\quad$ Community-centric services for easy creation of extranets, intranets, and social networks

$>\quad$ Familiar desktop UI and conventions

$>\quad$ Delegable granular security and role based authorizations

$>\quad$ Service Oriented Architecture (SOA) with web services support

$>\quad$ LDAP support and integration

$>\quad$ Portal as a Platform services for rapid portal development and deployment

$>\quad$ Secure enterprise application integration framework

Liferay Portal is a web platform with features commonly required for the development of websites and portals. Liferay includes a built-in web content management system allowing users to build websites and portals as an assembly of themes, pages, portlets/gadgets and a common navigation. ${ }^{[4]}$ Liferay is sometimes described as a content management framework or a web application framework. Liferay's support for plugins extends into multiple programming languages, including support for PHP and Ruby portlets.

\section{CONCLUSION}

Cloud computing is a major development in information technology, comparable in importance with the mainframe, the minicomputer, the microprocessor, and the interoperable.

Cloud computing is changing the way IT departments buy IT. Businesses have a range of paths to the cloud, including infrastructure, platforms and applications that are available from cloud providers as online services. Many people may be confused by the range of offerings and the terminology used to describe them and will be unsure of the risk and benefits there are many more players in the on-demand market that many reports acknowledge.

These range from basic infrastructure offerings (IAAS), through platform support (PAAS) to full applications (SAAS) .The long term cost of ownership may at first not seem to add up, but take into consideration factors such as reduced risk and added value and for many organizations on-demand services make a lot of sense.

\section{REFERENCES}

http://en.wikipedia.org/wiki/cloud_computing.

http://blog.appcore.com/blog/bid/167543/types-of-cloudcomputing-private-public-and-hybrid-clouds.

http://en.wikipedia.org/wiki/single_sign-on.

http://en.wikipedia.org/wiki/oauth.

http://en.wikipedia.org/wiki/openldap.

http://www.openldap.org/doc/admin24/guide.html

http://en.wikipedia.org/wiki/openldap.

http://en.wikipedia.org/wiki/replication_(computing).

https://cloudstack.apache.org/docs/en-

us/apache_cloudstack/4.2.0/html/admin_guide/whatis.html.

10. https://cloudstack.apache.org/docs/en-

us/apache_cloudstack/4.2.0/html/admin_guide/feature-

overview.html.

11. https://www.openshift.com/products/origin

12. http://en.wikipedia.org/wiki/openshift.

13. http://searchcloudapplications.techtarget.com/definition/openshift.

14. http://www.liferay.com/documentation/liferay-portal/6.1/userguide/-/ai/installing-liferay.

15. http://www.openldap.org/doc/admin22/install.html.

16. http://openshift.github.io/documentation/oo_deployment_guide_co mprehensive.html.

17. http://navinagarwalmca.wordpress.com/liferay-poratl-introductionand-basic.

18. http://en.wikipedia.org/wiki/liferay.

\section{BIOGRAPHY}

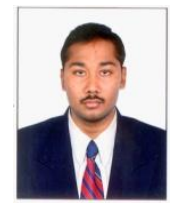

Karthik.b is the student of sri shakthi institute of engineering and technology in the Computer science and engineering department. Now I am doing my master of engineering course in final semester. 